\title{
A Theoretical Challenge: Transition-Metal Compounds
}

\author{
Markus Reiher*
}

\begin{abstract}
Quantum chemistry meets one of its greatest challenges in the field of transition-metal compounds capable of fascinating chemical transformations. Complicated electronic structure and interesting chemistry are intimately connected, and the theoretical elucidation of such molecular processes requires computational procedures that are both feasible and accurate. In this review we elaborate on our recent work in the field.
\end{abstract}

Keywords: Density matrix renormalization group $\cdot$ Relativistic quantum chemistry $\cdot$ Spin-spin coupling

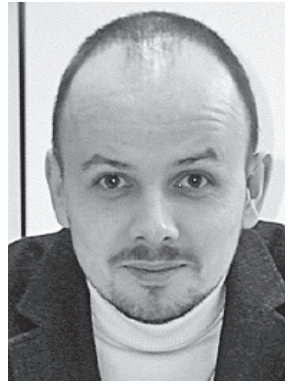

Markus Reiher was born in Paderborn (Westphalia) and obtained his $\mathrm{PhD}$ in theoretical chemistry in the group of Juergen Hinze in 1998. He completed his habilitation in theoretical chemistry at the University of ErlangenNuremberg in the group of Bernd Artur Hess in 2002. During this period he spent some time at Tel Aviv University, the KFKI Budapest, and the University of Tromso. From 2003 to 2005, he was Privatdozent at the University of Bonn and then moved to the University of Jena as a Professor for Physical Chemistry in 2005. Since the beginning of 2006 he has been Professor for Theoretical Chemistry at ETH Zürich.

Markus Reiher's research interests are diverse and cover many topics in molecular physics and chemistry.

\section{Introduction}

Quantum-chemical methods based on the first principles of quantum mechanics aim at the solution of the electronic Schrödinger equation,

$$
\hat{\mathrm{H}}^{e l} \Psi_{n}^{e l}=E_{n}^{e l} \Psi_{n}^{e l}
$$

This equation implies that we may calculate the electronic energy $E_{n}^{e l}$ from the electronic wave function $\Psi^{\text {el }}$ by means of the Hamiltonian operator $\hat{\mathrm{H}}^{e l}$, which contains operators for the kinetic energy of the electrons as well as for all electrostatic pair interaction energies of the electrons and the atomic nuclei in a molecule. The electronic energy depends on the positions $\boldsymbol{R}$ of all atomic nuclei, $E_{n}^{e l}=E_{n}^{e l}(\boldsymbol{R})$, and defines the potential energy hypersurface for chemical reactions. The index $n$ denotes the electronic state under consideration, and we are usually interested in the one with lowest energy, i.e. in the electronic ground state with $n=0$.

From the accurate solution of this equation follows the prediction of molecular structures (as minima on the electronic energy hypersurface), reaction energies (as differences of electronic energies calculated for specific molecules), and molecular properties of any kind (as electronic-energy derivatives). Hence, the above equation allows us to study molecular processes with as little experimental input as possible - only fundamental constants that enter $\hat{\mathrm{H}}^{e l}$ are needed. As a consequence, no model assumptions are made and original theoretical discoveries and predictions are possible. However, this ideal picture, which is in principle correct, is distorted by the fact that the interaction of electrons in molecules is difficult to describe. While few-electron atoms and molecules can be treated exactly in the so-called full config- uration interaction (FCI) approach, larger molecules require us to make approximations designed such that accuracy is compromised as little as possible. ${ }^{[1]}$

The history of theoretical transitionmetal chemistry is a history of approximations to the equation given above. Early approaches reduced the problem to Schrödinger-type equations which treat the central metal ion quantum mechanically perturbed by surrounding ligands modelled as partial charges (crystal-field theory) or in some more elaborate way (ligand-field theory).[2] Nowadays, these approximations are no longer necessary - though very helpful in order to better understand the fully quantum mechanical results - and the Schrödinger equation is solved for the full transition-metal complex including all ligands.

The quantum-chemical approach to transition-metal chemistry primarily aims at the prediction of

i) molecular structures of stable complexes and of transition states in reactions,

ii) the energies corresponding to these structures and

iii) their molecular properties in order to relate to experimental observations.

Because of the approximations intrinsic to quantum-chemical methods, all these observable quantities are affected by some error that needs to be assessed and then controlled. However, the method-inherent error is not the only error one has to deal with. In addition, a model error is introduced, which can, however, be well controlled. This error refers to the fact that one has to select a structural model for the transition-metal complex (and its potential reactants) which resembles the chemical process as closely as possible. In transition-metal chemistry, these structural models are usually well-defined since there is often only one metal center surrounded by well-structured ligands of less than, say, 200 atoms. The inclusion of environmental 
effects like interactions with solvent molecules can be decided on.

Fortunately, ligand binding energies are often large and dominated by the local reaction center, i.e. by the transition-metal center, so that dynamical effects may be neglected and a reaction mechanism can then be efficiently characterized and understood on the basis of only a few stationary structures on the potential energy hypersurface $E_{0}^{e l}(\boldsymbol{R})$. In other words, the reliable description of the electronic structure is often more important than the inclusion of the time-dependent motion of the reactants. In addition to the complicated electronic structure, which is characterized by dense lying electronic states $\Psi_{n}^{e l}$ usually due to the involvement of $d$-type orbitals, the open-shell nature of many transitionmetal complexes gives rise to states of different spin symmetry that must be taken into account.

In this review, we discuss some recent results from our work in computational transition-metal chemistry. We address the spin-state problem of density functional theory (DFT), the coupling of local spins in transition-metal clusters, the production of accurate reference data, and finally the calculation of molecular properties within the relativistic Douglas-Kroll-Hess theory. The paper concludes with a perspective on new developments that are in progress in our laboratory.

\section{Quantum Chemical Methods}

Historically, quantum chemical methods proved useful first for small closedshell molecules as they occur, for instance, in organic chemistry. For such molecules calculations of minimum structures within a single-determinant approximation for the electronic wave function of the ground state, $\Psi_{0}^{e l}$, often provide results of sufficient accuracy. In transition-metal chemistry, however, dense lying electronic states $\Psi_{n}^{e l}$ require wave-function models beyond the independent-particle approximation of Hartree-Fock theory. In principle, socalled (correlated) multi-determinant $a b$ initio methods - of which FCI is the most accurate one if a sufficiently large one-electron basis set is used - provide the optimal frame for reliable calculations on transition-metal compounds. However, such calculations are usually prohibitively expensive in terms of computational effort because of the unfortunate factorial scaling behavior of these methods with system size. In addition, the various approximations in correlated $a b$ initio models lead to different intrinsic deficiencies, which make their accuracy difficult to assess for transition-metal compounds. For instance, the complete-active-space self-consistent- field (CASSCF) approach, which implements the idea that a FCI-type wave function considering only the frontier orbitals in the complete active space (CAS) might be sufficient for describing the reactivity of a molecule, can produce qualitatively correct wave functions for transition-metal complexes. ${ }^{[3]}$ Its results can be improved by a subsequent second-order perturbation treatment (CASPT2) - needed to consider also those orbitals neglected from the CAS -, but the accuracy may still be limited due to the CAS reference being restricted for technical reasons to at most 18 electrons in 18 orbitals because of the factorially scaling computational effort required. By contrast, coupled-cluster calculations do not suffer from such CAS-limitations and have become very popular (especially in form of the so-called $\operatorname{CCSD}(\mathrm{T})$ variant which is regarded as some sort of standard model that guarantees accurate results). Such standard coupled-cluster methods are restricted to closed-shell reference wave functions. Open-shell transitionmetal complexes, however, require a multireference coupled-cluster scheme. Such schemes are currently being developed by various groups but one may estimate on the basis of the enormous effort required for a standard single-reference coupled-cluster model like $\operatorname{CCSD}(\mathrm{T})$, which formally scales like $O\left(M^{7}\right)$ with system size $M($ e.g. number of one-electron basis functions), that multi-reference coupled-cluster calculations will be feasible only for comparatively small molecules (with much less than a dozen atoms). Efforts in many research groups aim at implementations of ab initio methods that eventually scale linearly with increasing system size. ${ }^{[4]}$ However, these linear-scaling methods perform best if the molecule under consideration features an extended structure built up by well-defined subunits (as in the case of a polymer).

Because of the limitations of the highly accurate $a b$ initio approaches indicated above, the dawn of computational transition-metal chemistry coincides with the advent of gradient-corrected density functionals introduced to DFT about twenty years ago (many review articles exist; see for examples refs. [5]). Despite the fact that these density functionals are employed in a single-determinant, the so-called KohnSham framework, which is algorithmically very much alike the standard Hartree-Fock protocol, the results obtained were surprisingly accurate. The rise of DFT was then followed by the identification of DFT failures, which have been reported since the late 1990s. Though in principle exact, contemporary DFT in the framework established by Kohn and Sham is hampered by the present-day approximations for the exchange-correlation functional.[6] Especially the past few years have seen new ad- vances in the development of functionals, as for example, provided by orbital-dependent functionals (see e.g. refs. [7]) or by exchange-correlation density functionals with position-dependent mixing functions for the combination of different functional expressions. ${ }^{[8]}$ Also, new functionals parametrized on a well-balanced set of reference data have been devised ${ }^{[9]}$ to mention only a few of the various promising recent developments.

Apart from the approximation chosen for the electronic wave function - or for the exchange-correlation density functional - also the Hamiltonian operator in Schrödinger's equation requires some attention. The Hamiltonian is exactly known in nonrelativistic Schrödinger quantum mechanics, which is an approximate description of physical reality as it does not obey the principles of Einstein's theory of special relativity since an infinite speed of light is assumed. ${ }^{[10]}$ As a result, kinematic relativistic effects and spin-orbit coupling are completely neglected. It is important to understand that a consistent treatment of relativistic effects is the more important the higher the nuclear charge of the metal atom is. Hence, these effects must not be neglected in transition-metal chemistry.

A consistent relativistic description can be based on the Dirac one-electron Hamiltonian and yields the so-called fourcomponent methods. ${ }^{[10]}$ However, routine calculations are involved and very expensive in terms of computing time. For instance, a four-component $a b$ initio correlated calculation on a metal dimer like $\mathrm{Hg}_{2}$ or $\mathrm{U}_{2}$ with a sufficiently large basis set requires significant computer resources. Therefore, quasi-relativistic one- and twocomponent methods emerged of which the most prominent ones are the simple and efficient zeroth-order regular approximation (ZORA) ${ }^{[11]}$ and the formally elegant Douglas-Kroll-Hess (DKH) transformation theory, ${ }^{[12]}$ which have attracted considerable attention in the new millennium. Also, relativistic effective core potentials ${ }^{[13]}$ are heavily used although their accuracy and applicability can be limited if, for instance, molecular properties probed at an atomic nucleus are desired.

\section{The Spin-State Problem}

It is important to note that the DFT approach to large transition-metal complexes is currently the only feasible one if their chemistry shall be routinely investigated. Even if special variants of $a b$ initio methods are feasible for a certain molecule, the molecule's structure is likely to be optimized in a DFT calculation under the assumption that the molecular structure will be reliable even if the corresponding DFT 
energy is not. Therefore, the assessment of DFT methods in transition-metal chemistry is decisive. One systematic failure of DFT of particular importance in transition-metal chemistry is the spin-state energetics problem. ${ }^{[14]}$ The reliable calculation of states of different spin is crucial - especially for the correct prediction of reaction mechanisms (cf. refs. [15] for two examples). This is particularly true if a change of spin in the course of a reaction represents an essential step within the reaction mechanism (see the two-state reactivity concept by Schröder, Schwarz and Shaik $\left.{ }^{[16]}\right)$.

One focus of our research has been the assessment, reparametrization and application of density functional theory for the spin-state problem in transition-metal chemistry. We encountered the need to reliably calculate energies of different spin states first in the context of iron-sulfur complexes in 2001 and discovered a systematic linear dependence of spin-state energy differences on the admixture of exact (Hartree-Fock-type) exchange in the density functional. ${ }^{[17]}$ This systematic dependence revealed that the main difference between various (approximate) density functionals is the admixture of exact exchange. Pure density functionals turned out to yield very similar spin-state energy splittings compared to those obtained from hybrid functionals. The deviation between pure and hybrid functionals in this respect can amount to more than $100 \mathrm{~kJ} / \mathrm{mol}$, which makes the calculation of reaction mechanisms very difficult - especially if two-state reactivity is important. The importance of accurately calculated spin-state energetics can hardly be overemphasized since they play a prominent role in catalysis and in bioinorganic chemistry.

Discovered first for iron-sulfur complexes, ${ }^{[17]}$ which have become some sort of standard test molecules for the assessment of quantum-chemical methods, ${ }^{[18]}$ we soon investigated a series of metallocenes and metallabenzenes ${ }^{[19]}$ and confirmed that a reparametrized B3LYP density functional with reduced exact-exchange admixture (15\% instead of $20 \%$ ), which we dubbed B3LYP*, can consistently improve on spinstate energetics at equilibrium structures. Despite the simple nature of this reparametrization of the functional, which is clearly no satisfactory long-term solution but only a pragmatic one especially since it is not clear whether the calculated spin densities are reliable, ${ }^{[20]}$ the effect is decisive and stimulated work ${ }^{[21]}$ on the most critical cases, namely on spincrossover compounds, [22] which show a thermally induced spin flip and possess a high-spin-low-spin energy splitting of the size of the thermal energy $R T$. Even for these complexes we obtained quite satisfactory results, which allowed us to predict the likelihood of thermal spin- crossover based on the electronic-energy gap between high-spin and low-spin states. In turn, we were able to explain previous results on spincrossover complexes. ${ }^{[23]}$ All these studies rest on comparisons of different density functionals because truly reliable $a b$ initio reference data are very difficult to obtain (see also below). However, for a resilient development of new density functionals more theoretical work on their functional form will be necessary.

\section{Local Spins}

The spin-state problem described above for mononuclear transition-metal complexes becomes even more severe when electronic spin-spin interactions in polynuclear clusters need to be considered. Such clusters are, for instance, found in active sites of metalloenzymes and often feature interesting physical and chemical properties as molecular magnets and catalysts. In these cases the accurate calculation of two electronic energy levels, namely those of the ferromagnetically coupled high-spin state and the antiferromagnetically coupled low-spin state, determines the coupling strength $J$ of two (artificial) electronic spins located at fragments in such a spin-spin-interacting cluster. The coupling constant $J$ is extracted from these two energies according to the phenomenological Heisenberg Hamiltonian ${ }^{[24]}$ and facilitates a direct comparison with experimentally derived coupling strengths.

The Heisenberg Hamiltonian connects the coupling constant $J$ with the product of two electronic spin operators assigned to the interacting metal centers. Hence, it is of the form of an operator that describes the quantum-mechanical coupling (interaction) of angular momenta and is therefore also called magnetic coupling. However, it is by far not obvious how a spin operator assigned to some position or basin in space (i.e. to an atom in a molecule) can be extracted from the individual spin operators of the electrons in the cluster because they are not bound to specific atoms. Though a definition of local spins is always affected by an anthropogenic choice of the definition of the local basin, this choice has still to be made in the most consistent way possible. Clark and Davidson[25] elaborated on a choice defined by standard atomcentered one-electron basis sets which we investigated in some detail. ${ }^{[26]}$ A different choice based on a spatial decomposition of spin expectation values was then suggested by Mayer to eliminate the nonvanishing local Clark and Davidson spins in closedshell molecular structures. ${ }^{[27]}$ In the first implementation of the Mayer scheme, ${ }^{[28]}$ we could demonstrate that the Mayer local spins agree well with idealized local spin quantum numbers (e.g. one would obtain a Mayer local spin for iron of about $S_{\mathrm{Fe}}=2$ for a high-spin Fe(II) complex).

DFT calculations of Heisenberg coupling constants $J$ are complicated by the need to converge the so-called broken(spin-)symmetry determinant as an approximation for the antiferromagnetically coupled state.[29] Van Voorhis and collaborators ${ }^{[30]}$ proposed a controlled convergence scheme based on a Lagrangian multiplier technique. We have investigated this approach, which is incredibly useful in routine quantum-chemical studies on transition-metal clusters, and have elaborated on a possibility to soften the idealized-spin condition of the original approach within a restrained algorithm. ${ }^{[31]}$

Despite the huge number of papers dedicated to broken-symmetry DFT calculations on such clusters, almost no systematic investigation of different density functionals in broken-symmetry calculations existed. Therefore, we investigated a dinuclear bis- $\mu$-oxo-iron cluster with density functionals of varying exact-exchange admixture. ${ }^{[32]}$ Again, a systematic dependence of energy splittings and Heisenberg coupling constants on exact-exchange admixture in the functional was found.

\section{DMRG for Accurate Reference Data}

In order to assess and improve on the accuracy of contemporary density functionals one strategy to follow is to aim at highly accurate $a b$ initio reference data for comparison with DFT results. Examples have been given by many groups (see refs. [33] to mention only a few), where configuration-interaction-type methods like CASSCF, CCSD(T), or multireference $\mathrm{CI}$ have been employed. The basic idea of any such configuration-interaction-like approach is to include all important electronic configurations, i.e. Slater determinants $\Psi_{1}(N)$, into the $N$-electron basis set expansion of the total wave function

$$
\Psi_{n}^{e l}(N)=\sum_{I=1}^{m} C_{I}^{(n)} \Phi_{I}(N)
$$

where $N$ is the total number of electrons in the molecule under consideration and $m$ denotes the number of $N$-electron basis functions $\Phi_{1}(N)$, which is infinite in the case of an exact solution. The CI coefficients $C_{I}^{(n)}$ are determined by solution of an eigenvalue problem.

Unfortunately, decades of intense work in theoretical chemistry have shown that incredibly large CI expansions are required if an accuracy of about $1 \mathrm{kcal} / \mathrm{mol}$ is desired for relative energies like reaction energies. 
Usually, these methods converge the absolute energy of a molecule and achieve this goal either by an efficient basis-set expansion as in the case of the coupled-cluster models or by a pre-selection of dominant configurations before a standard orbital substitution pattern (usually called excitation hierarchy) is employed as in a multireference $\mathrm{CI}$ approach. Of course, spending a tremendous effort on the accurate calculation of the absolute energy might not always be necessary. Consequently, tailored (difference-dedicated) CI approaches ${ }^{[34]}$ aim at the accurate calculation of energy differences with less effort.

The main feature of all these approaches is that the analytic expansion of the electronic wave function given above, from which the electronic energy is calculated, is pre-defined. Surely, one may always include a new or skip an old configuration (determinant) in the basis set expansion according to some rule of thumb, but the energy is always obtained for an expansion defined prior to the calculation of its parameters. However, a quite novel CI-type method has become available, which can be beneficial in transition-metal chemistry as we have demonstrated recently. ${ }^{[35]}$ This method is called the density matrix renormalization group (DMRG) and was invented by White ${ }^{[36]}$ based on earlier work by Wilson. ${ }^{[37]}$ Introduced to solid-state physics in the 1990s, several groups pioneered the application of DMRG in a chemical context. [38]

DMRG is an iterative, adaptive approach to expand the electronic wave function into an $N$-electron basis whose composition changes until convergence is reached (for details of the algorithm we may refer to refs. [39]). The basis functions are most general FCI-type functions with weights (CI-like coefficients) that are determined in the DMRG iterations. The explicit analytic composition of this $N$-electron basis can in practice only be known for the smallest molecules ${ }^{[39 \mathrm{~d}]}$ (for the same reason for that FCI is only feasible for such small molecules). However, this is no drawback as the electronic energy is obtained by diagonalization of the optimized matrix representation of the Hamiltonian. Of course, the convergence and scaling behavior of the DMRG algorithm depends on DMRG-specific parameters, but as in any other approximate CI-type approach one may hope that the target quantities (mostly the electronic energies) converge fast with an efficient choice of these parameters.

And indeed, we could demonstrate that spin-state energy differences as well as energy differences between different molecular structures of transition-metal clusters $^{[35]}$ are quite reliably obtained in DMRG calculations. Accordingly, DMRG has the potential to substitute CASSCF ap- proaches if large active orbital spaces are required so that $m$ in the equation given in this section above would be too large for actual calculations. Hence, DMRG is a particularly promising approach for calculations on metal complexes with large unsaturated ligands (like porphyrins) or with noninnocent ligands and for polynuclear transition-metal clusters. We also observed a behavior of DMRG to select those configurations $\Phi_{1}(N)$ that are decisive for relative electronic energies, which is thus comparable to the difference-dedicated CI approach, ${ }^{[35]}$ but now in a completely automated and in principle exact manner. Here, the flexibility of DMRG to produce a truly optimum $N$-electron basis set for the wave-function expansion with hardly any restrictions is highlighted when compared to the pre-defined form of analytic basis expansions in standard CI procedures.

\section{Douglas-Kroll-Hess Theory and Relativistic Effects}

As already mentioned in the beginning of this review, the sufficiently accurate description of the electronic structure is only one part of the theoretical challenge of transition-metal chemistry. Especially heavy metal atoms require a Hamiltonian that is in accordance with the theory of relativity. The relativistic theory of the electron rests on Dirac's equation that replaces the nonrelativistic Schrödinger equation. The difference between results from a consistent relativistic calculation and one based on standard Schrödinger quantum mechanics is called relativistic effect. Such effects are often discussed in terms of orbital contractions that eventually affect the electron density and hence the chemistry of transition-metal complexes. ${ }^{[40]}$

In the past years, one focus of our work in the development of relativistic quantumchemical methods was on the DKH theory, in which a sequence of unitary transformations is applied to the Dirac Hamiltonian in order to decouple the desired positiveenergy ('electronic') states from the negative-energy ones, i.e. from the so-called positronic states that are not relevant in chemical investigations but increase the computational effort. Stimulated by work of Nakajima and Hirao, ${ }^{[41]}$ who presented the first DKH results of third order in the external potential, we revisited and largely extended the DKH method. ${ }^{[42]}$ Arbitraryorder generalized DKH energy calculations have become feasible ${ }^{[43]}$ and our new arbitrary-order scalar relativistic DKH implementation is designed to be strictly modular so that it can be easily combined with standard quantum chemistry program packages. Recent developments ${ }^{[44]}$ then reduced the computational effort also for four-component methods by the introduction of ideas first derived for two-component approaches like ZORA and DKH.

Also the calculation of various molecular properties in the DKH framework has been implemented in a most general way. ${ }^{[45]}$ Detailed studies of the approach for electric-field-like properties ${ }^{[46]}$ then allowed us to work on the electron density at an atomic nucleus and on the electric field gradient, ${ }^{[47]}$ which both enter the theory of Moessbauer spectroscopic parameters. Current work in our laboratory aims at the establishment of a rigorous, reliable and transferable protocol for the calculation of Moessbauer isomer shifts for iron complexes.

Of course, also magnetic properties are important as diagnostic tools in transitionmetal chemistry. These, however, are not easy to implement in a DKH framework. ${ }^{[48]}$

\section{Conclusion and Outlook}

Present-day quantum-chemical methods allow us to study and to predict reactions at transition-metal complexes where all reactants are described quantum mechanically in contrast to crystaland ligand-field theories, which treat the metal-center surroundings in a much more approximate way. However, the average accuracy obtained for electronic energy differences (excitation energies, reaction energies) is still not sufficient for original predictions, and hence a close comparison to experimental data is always necessary where possible. Current quantum chemical approaches - especially DFT methods - live on the fact that the decision of certain chemical questions of structure or of relative reaction energies can afford comparatively large errors in the calculated results.

In the long run, however, a systematic error theory is needed which connects a scientific assertion based on computational results with error bars assigned to the calculation in order to guarantee the validity of the calculated results. Currently, this aspect is often reduced to the claim that a quantum-chemical method should be systematically improvable in order to guarantee a protocol for the improvement of calculated results once they turn out to be not accurate enough. This feature is completely absent in all DFT methods as there exists no recipe on how to systematically improve on an approximate density functional employed in the calculations. Furthermore, the situation is difficult because the individual error in a DFT calculation on some complex may be quite large although the average error with that functional may be acceptable; and it is hard if not impossible to tell when the 
individual error becomes large compared to the average error (determined for a test molecule set). By contrast, $a b$ initio methods may always be improved by increasing the $\mathrm{N}$-electron basis-set size (e.g. via higher 'excitations'). However, systematic improvability is not sufficient for arriving at accurate results since procedures need to be found that increase the basis-set size in a most efficient way in order not to collect a lot of deadwood with little effect on the desired physical quantities.

All these difficulties of improving the computational models go by the name of solving the electron-correlation problem, and therefore methodological developments are still at the heart of research in quantum chemistry. But research in this direction is not only concerned with methodological and algorithmic advances, also theoretical work is still needed and in progress. One example of the latter is the role of electron spin in DFT. ${ }^{[49]}$ In the focus of our current work is the spatial resolution of deviations found with contemporary approximate density functionals when compared with one another ${ }^{[50]}$ and with the exact density functional reconstructed from accurate densities and spin-densities.

For a true de novo design of transitionmetal complexes, especially if they shall serve as functional units (e.g. as catalysts), an inverse approach to quantum chemistry[51] is necessary, which denotes the re-formulation of well-known quantumchemical equations in such a way that the formalism provides a direct answer to a given scientific question. We have already demonstrated how this can be achieved for molecular vibrations if one is looking for the properties of a specific molecular vibration (mode-tracking) ${ }^{[52]}$ or if the distribution of spectroscopic intensities on molecular distortions is sought (intensity-tracking) to directly yield all intense vibrations in a spectrum. ${ }^{[53]}$ We are currently working on extensions to other properties like energies and structures. In computational drug and materials design this is known as the rational exploration of chemical space and we may refer to two interesting examples in these areas. ${ }^{[54]}$ Only work along these lines will eventually replace the still needed chemical intuition - i.e. the profound knowledge of the experimentally working chemists - in the design of chemical reactions and reactants. Purpose-driven algorithms like mode-tracking represent a new generation of quantum chemical methods. More of them will surely become available because of the tremendously increased computational power of desktop computers that allows us to loosen the corset of the efficiency paradigm which governs the derivation and implementation of quantum-chemical equations solely focused on their fast solution for ever-increasing mo- lecular sizes. Even new concepts for the explorations of chemical reactivity - like the possibility to physically experience reactivity in a haptic-quantum-chemistry set-up ${ }^{[55]}$ - become now feasible and allow us to push the limits of computational transition-metal chemistry forward.

In view of the remarkable achievements regarding structures, energies, and (spectroscopic) properties achieved worldwide, we believe that the ultimate goal in theoretical transition-metal chemistry will become the de novo design of transitionmetal catalysts via the direct, theory-based prediction of metal complex structures (including the rigorous theoretical determination of optimal central metal ion(s) and the design of chelate-ligand or nano-structured surroundings). Work along this paradigm is in progress in our laboratory.

\section{Acknowledgments}

The work reviewed here was supported by the Schweizer Nationalfonds (200020-121870), by the ETH Zürich (grant TH-26 07-3) and by the German Science Foundation DFG (projects $\operatorname{Re} 1703 / 1-1,2)$.

\section{Received: January 15, 2009}

[1] T. Helgaker, P. Jørgensen, J. Olsen, 'Molecular Electronic-Structure Theory' John Wiley \& Sons, Chichester, England, 2000.

[2] J. S. Griffith, 'The Theory of Transition-Metal Ions' Cambridge University Press, Cambridge, 1964.

[3] L. Gagliardi, B. O. Roos, Chem. Soc. Rev. 2007, 36, 893 .

[4] a) C. Ochsenfeld, J. Kussmann, D. S. Lambrecht, Rev. Comput. Chem. 2007, 23, 1; b) S. Goedecker, Rev. Mod. Phys 1999, 71, 1085.

[5] a) B. Delley, M. Wrinn, H. P. Lüthi, J. Chem. Phys. 1994, 100, 5785; b) T. Ziegler, Can. J. Chem. 1995, 73, 743; c) P. E. M. Siegbahn, 'Electronic Structure Calculations for Molecules Containing Transition Metals', in 'Advances in Chemical Physics', Vol. 43 , Eds. I. Prigogine, S. A. Rice, John Wiley \& Sons, Inc., 1996, pp. 333-387; d) T. Ziegler, 'Density-functional Theory as a Practical Tool in Studies of Transition Metal Chemistry and Catalysis', in 'Density-functional Methods in Chemistry and Materials Science', Ed. M. Springborg, John Wiley \& Sons, Inc., 1997, pp. 69-103; e) C. W. Bauschlicher, Jr., A. Ricca, H. Partridge, 'Chemistry by Density Functional Theory', in 'Recent Advances in Density Functional Methods', Vol. 2, Ed. D. P. Chong, World Scientific, Singapore, 1997, Chapter 6, pp. 165-227; f) H. Chermette, Coord. Chem. Rev. 1998, 178-180, 699; g) G. Frenking, T. Wagener, 'Transition Metal Chemistry', in 'Encyclopedia of Computational Chemistry', Ed. P. v. R. Schleyer, John Wiley \& Sons, Chichester, 1998, pp. 3073-3084; h) A. Görling, S. B. Trickey, P. Gisdakis, N. Rösch, 'A Critical Assessment of Density Functional Theory with Regard to Applications in Organometallic Chemistry', in 'Topics in Organometallic Chemistry', Vol. 4, Eds. J. M. Brown, P. Hofmann, SpringerVerlag, Berlin, Heidelberg, 1999, pp. 109-163; i) S. Niu, M. B. Hall, Chem. Rev. 2000, 100, 353; j) P. E. M. Siegbahn, M. R. A. Blomberg, Chem. Rev. 2000, 100, 421; k) G. Frenking, N. Fröhlich, Chem. Rev. 2000, 100, 717; 1) T. R. Cundari, Chem. Rev. 2000,
100, 807; m) W. Koch, M. C. Holthausen, 'A Chemist's Guide to Density Functional Theory', Wiley-VCH, Weinheim, 2000; n) K. Pierloot, in 'Computational Organometallic Chemistry', Ed. T. R. Cundari, Marcel Dekker, New York, 2001; o) M. Diedenhofen, T. M. Wagener, G. Frenking, 'The Accuracy of Quantum Chemical Methods for the Calculation of Transition Metal Compounds' in 'Computational Organometallic Chemistry', Ed. T. R. Cundari, Marcel Dekker, New York, 2001; p) T. Ziegler, J. Chem. Soc., Dalton Trans. 2002, 642; q) T. Ziegler, J. Autschbach, Chem. Rev. 2005 105, 2695; r) B. Kirchner, F. Wennmohs, S. Ye, F. Neese, Current. Opin. Chem. Biol. 2007, 11, $134 ;$ s) F. Neese, T. Petrenko, D. Ganyushin, G. Olbrich, Coord. Chem. Rev. 2007, 251, 288.

[6] R. G. Parr, W. Yang, 'Density-Functional Theory of Atoms and Molecules', in 'International Series of Monographs on Chemistry', Vol. 16, Eds. R. Breslow, J. B. Goodenough, J. Halpern, J. S. Rowlinson, Oxford Science Publications, New York, 1989

[7] a) A. Görling, J. Chem. Phys. 2005, 123, 062203; b) E. J. Baerends, O. V. Gritsenko, J. Chem. Phys. 2005, 123, 062202.

[8] a) J. Tao, J. P. Perdew, V. N. Staroverov, G. E. Scuseria, Phys. Rev. Lett. 2003, 91, 146401; b) M. Kaupp, H. Bahmann, A. V. Arbuznikov, J. Chem. Phys. 2007, 127, 194192.

[9] Y. Zhao, D. Truhlar, Theor. Chem. Acc. 2008, 120, 215.

[10] M. Reiher, A. Wolf, 'Relativistic Quantum Chemistry', Wiley-VCH, Weinheim, 2009

[11] a) E. van Lenthe, E. J. Baerends, J. G. Snijders, J. Chem. Phys. 1993, 99, 4597; b) E. van Lenthe, E. J. Baerends, J. G. Snijders, J. Chem. Phys. 1994, 101, 9783

[12] a) M. Douglas, N. M. Kroll, Ann. Phys. 1974, 82, 89; b) B. A. Hess, Phys. Rev. A 1986, 33, 3742 .

[13] M. Dolg, 'Relativistic Effective Core Potentials', in 'Relativistic Quantum Chemistry - Vol I. Theory', Ed. P. Schwerdtfeger, Elsevier, 2002, pp. 793-862.

[14] a) J. N. Harvey, M. Aschi, Faraday Discuss. 2003, 124, 129; b) R Poli, J. N. Harvey, Chem. Soc. Rev. 2003, 32, 1 .

[15] a) D. W. Keogh, R. Poli, J. Am. Chem. Soc. 1997, 119, 2516; b) M. Filatov, S. Shaik, J. Phys. Chem. A 1998, 102, 3835.

[16] D. Schröder, S. Shaik, H. Schwarz, Acc. Chem. Res. 2000, 33, 139.

[17] M. Reiher, O. Salomon, B. A. Hess, Theor Chem. Acc. 2001, 107, 48.

[18] a) K. Pierloot, S. Vancoillie, J. Chem. Phys. 2008, 128, 034104; b) F. Aquilante, T. B. Pedersen, R. Lindh, J. Chem. Phys. 2007, 126, 194106.

[19] O. Salomon, M. Reiher, B. A. Hess, J. Chem. Phys. 2002, 117, 4729.

[20] J. Conradie, A. Ghosh, J. Phys. Chem. B 2007, 111,12621

[21] M. Reiher, Inorg. Chem. 2002, 41, 6928.

[22] a) P. Gütlich, Struct. Bond. 1981, 44, 83; b) P. Gütlich, A. Hauser, H. Spiering, Angew. Chem. 1994, 106, 2109.

[23] H. Paulsen, L. Duelund, H. Winkler, H. Toftlund, A. X. Trautwein, Inorg. Chem. 2001, 40, 2201.

[24] a) P. A. M. Dirac, Proc. R. Soc. London, Ser. A 1926, 112, 661; b) P. A. M. Dirac, Proc. R. Soc. London, Ser. A 1929, 123, 714; c) W. Heisenberg, Z. Phys. 1926, 38, 411; d) W. Heisenberg, Z. Phys. 1928, 49, 619.

[25] A. E. Clark, E. R. Davidson, J. Chem. Phys. 2001, 115, 7382

[26] C. Herrmann, M. Reiher, B. A. Hess, J. Chem. Phys. 2005, 122, 034102.

[27] I. Mayer, Chem. Phys. Lett. 2007, 440, 357.

[28] M. Podewitz, C. Herrmann, A. Malassa, M. Westerhausen, M. Reiher, Chem. Phys. Lett. 2008, 451,301 
[29] a) L. Noodleman, J. Chem. Phys. 1981, 74 5737; b) L. Noodleman, E. R. Davidson, Chem. Phys. 1986, 109, 131; c) L. Noodleman, D. A. Case, Adv. Inorg. Chem. 1992, 38, 423 d) L. Noodleman, C. Y. Peng, D. A. Case, J. M. Mouesca, Coord. Chem. Rev. 1995, 144 , 199.

[30] I. Rudra, Q. Wu, T. V. Voorhis, J. Chem. Phys. 2006, 124, 024103 .

[31] C. Herrmann, M. Podewitz, M. Reiher, J. Comput. Chem. 2009, doi: 10.1002/qua.21997.

[32] C. Herrmann, L. Yu, M. Reiher, J. Comput. Chem. 2006, 27, 1223.

[33] a) R. Poli, J. N. Harvey, Chem. Soc. Rev. 2003 , 32, 1; b) J. N. Harvey, Struct. Bond. 2004, 112 , 151 ; c) A. Fouqueau, S. Mer, M. E. Casida, L. M. Lawson Daku, A. Hauser, T. Mineva, F. Neese, J. Chem. Phys. 2004, 120, 9473; d) L. M. Lawson Daku, A. Vargas, A. Hauser, A. Fouqueau, M. E. Casida, ChemPhysChem 2005, 6, 1393; e) G. Ganzenmüller, N. Berkaine, A. Fouqueau, M. E. Casida, M. Reiher, J. Chem. Phys. 2005, 122, 234321; f) A. Fouqueau, M. E. Casida, L. M. Lawson Daku, A. Hauser F. Neese, J. Chem. Phys. 2005, 122, 044110 g) G. Frenking, M. Sola, S. F. Vyboishchikov, J. Organomet. Chem. 2005, 690, 6178, h) W. Petz, C. Kutschera, M. Heitbaum, G. Frenking, R. Tonner, B. Neumüller, Inorg. Chem. 2005, 44, 1263; i) M. Hölscher W. Leitner, M. C. Holthausen, G. Frenking, Chem. Eur. J. 2005, 11, 4700; j) M. F. Rode, H.-J. Werner, Theor. Chem. Acc. 2005, 114 309; k) C. J. Cramer, M. Wloch, P. Pecuch, C. Puzzarini, L. Gagliardi, J. Phys. Chem. A 2006, 110, 1991.

[34] a) O. Castell, R. Caballol, J. P. Malrieu, Chem. Phys. 1993, 172, 33; b) F. Neese, J. Chem. Phys. 2003, 119, 9428

[35] K. H. Marti, I. Malkin Ondik, G. Moritz, M. Reiher, J. Chem. Phys. 2008, 128, 014104.

[36] a) S. R. White, Phys. Rev. Lett. 1992, 69, 2863 ; b) S. R. White, Phys. Rev. B 1993, 48, 10345.

[37] K. G. Wilson, Rev. Mod. Phys. 1983, 55, 583
[38] a) G. Fano, F. Ortolani, L. Ziosi, J. Chem. Phys. 1998, 108, 9246; b) S. R. White, R. L. Martin, J. Chem. Phys. 1999, 110, 4127; c) S. Daul, I. Ciofini, C. Daul, S. R. White, Int. J. Quantum Chem. 2000, 79, 331; e) A. O. Mitrushenkov, G. Fano, F. Ortolani, R. Linguerri, P. Palmieri, J. Chem. Phys. 2001, 115, 6815; f) A. O. Mitrushenkov, R. Linguerri, P. Palmieri, G. Fano, J. Chem. Phys. 2003, 119, 4148; g) Ö. Legeza, J. Röder, B. A. Hess, Phys. Rev. B 2003, 67, 125114; h) Ö. Legeza, J. Röder, B. A. Hess, Mol. Phys. 2003, 101 , 2019; i) G. K.-L. Chan, M. Head-Gordon, J. Chem. Phys. 2003, 118, 8551; j) G. K.-L. Chan, M. Kallay, J. Gauss, J. Chem. Phys. 2004, 121, 6110 ; k) G. Moritz, B. A. Hess, M. Reiher, $J$. Chem. Phys. 2005, 122, 024107; 1) G. Moritz, A. Wolf, M. Reiher, J. Chem. Phys. 2005, 123, 184105; m) G. Moritz, M. Reiher, J. Chem. Phys. 2006, 124, 034103; n) J. Hachmann, W. Cardoen, G. K.-L. Chan, J. Chem. Phys. 2006, 125, 141101; o) J. Hachmann, J. J. Dorando, M. Avilés, G. K.-L. Chan, J. Chem. Phys. 2007, 127, 134309; p) D. Ghosh, J. Hachmann, T. Yanai, G. K.-L. Chan, J. Chem. Phys. 2008, 128, 144117; q) D. Zgid, M. Nooijen, J. Chem. Phys. 2008, 128, 014107.

[39] a) G. K.-L. Chan, M. Head-Gordon, J. Chem. Phys. 2002, 116, 4462; b) G. K.-L. Chan, J. Chem. Phys. 2004, 120, 3172; c) G. K.L. Chan, T. Van Voorhis, J. Chem. Phys. 2005 , 122, 204101; d) G. Moritz, M. Reiher, J. Chem. Phys. 2007, 126, 244109; e) D. Zgid, M. Nooijen, J. Chem. Phys. 2008, 128, 144116; f) D. Zgid, M. Nooijen, J. Chem. Phys. 2008, 128,144115 .

[40] a) G. Eickerling, R. Mastalerz, V. Herz, H.J. Himmel, W. Scherer, M. Reiher, J. Chem. Theory Comput. 2007, 3, 2182; b) N. Hebben, H.-J. Himmel, G. Eickerling, C. Herrmann, M. Reiher, V. Herz, M. Presnitz, W. Scherer, Chem. Eur. J. 2007, 13, 10078.

[41] T. Nakajima, K. Hirao, J. Chem. Phys. 2000, 113,7786 .
[42] a) A. Wolf, M. Reiher, B. A. Hess, J. Chem. Phys. 2002, 117, 9215; b) M. Reiher, A. Wolf J. Chem. Phys. 2004, 121, 2037.

[43] M. Reiher, A. Wolf, J. Chem. Phys. 2004, 121 10945.

[44] a) W. Kutzelnigg, W. Liu, J. Chem. Phys. 2005, 123, 241102; b) M. Filatov, J. Chem. Phys. 2006, 125, 107101; c) M. Ilias, T. Saue, J. Chem. Phys. 2007, 126, 064102.

[45] A. Wolf, M. Reiher, J. Chem. Phys. 2006, 124, 064102.

[46] A. Wolf, M. Reiher, J. Chem. Phys. 2005, 124, 064103.

[47] a) R. Mastalerz, G. Barone, R. Lindh, M. Reiher, J. Chem. Phys. 2007, 127, 074105 ; b) R. Mastalerz, R. Lindh, M. Reiher, Chem. Phys. Lett. 2008, 465, 157.

[48] S. Luber, I. Malkin Ondik, M. Reiher, Chem. Phys. 2009; 365, 205.

[49] M. Reiher, Faraday Discuss. 2007, 135, 97.

[50] a) K. Kiewisch, G. Eickerling, M. Reiher, J. Neugebauer, J. Chem. Phys. 2008, 128, 044114; b) S. Fux, K. Kiewisch, C. R. Jacob, J. Neugebauer, M. Reiher, Chem. Phys. Lett 2008, 461, 353; c) M. Reiher, K. Meier, J. Neugebauer, 2009, in preparation.

[51] a) E. A. Carter, P. J. Rossky, Acc. Chem. Res. 2006, 39, 71; b) C. Herrmann, J. Neugebauer, M. Reiher, New J. Chem. 2007, 31, 818.

[52] M. Reiher, J. Neugebauer, J. Chem. Phys. 2003 $118,1634$.

[53] a) K. Kiewisch, J. Neugebauer, M. Reiher, J. Chem. Phys. 2008, 129, 204103; b) S. Luber, J. Neugebauer, M. Reiher, J. Chem. Phys. 2009, 130, 064105

[54] a) A. V. Lilienfeld, R. D. Lins, U. Röthlisberger, Phys. Rev. Lett. 2005, 95, 153002; b) M. Wang, X. Hu, D. N. Beratan, W. Yang, J. Am. Chem. Soc. 2006, 128, 3228.

[55] K. H. Marti, M. Reiher, J. Comput. Chem. 2009; doi:10.1002/jcc. 21201 . 